\title{
Dosimetric comparison between two MLC systems commonly used for stereotactic radiosurgery and radiotherapy: A Monte Carlo and experimental study
}

\author{
K. Asnaashari ${ }^{\text {a }}$, James C.L. Chow ${ }^{\text {b,c,d, }}{ }^{\text {, Mostafa Heydarian }}{ }^{\text {b,c }}$ \\ a Department of Technology of Radiology and Radiotherapy, Allied Medical Science School, \\ Tehran University of Medical Science, Tehran, Iran \\ ${ }^{\mathrm{b}}$ Department of Radiation Physics, Princess Margaret Hospital, Toronto, ON M5G 2M9, Canada \\ ' Department of Radiation Oncology, University of Toronto, Toronto, ON M5G 2M9, Canada \\ ${ }^{\mathrm{d}}$ Department of Physics, Ryerson University, Toronto, ON M5B 2K3, Canada
}

Received 27 November 2011; received in revised form 24 April 2012; accepted 7 May 2012 Available online 31 May 2012

\author{
KEYWORDS \\ Stereotactic \\ radiosurgery; \\ Monte Carlo; \\ Dosimetry; \\ Multi-leaf collimator; \\ Radionics
}

\begin{abstract}
In this work dosimetric parameters of two multi-leaf collimator (MLC) systems, namely the beam modulator (BM), which is the MLC commercial name for Elekta "Synergy $S$ " linear accelerator and Radionics micro-MLC (MMLC), are compared using measurements and Monte Carlo simulations. Dosimetric parameters, such as percentage depth doses (PDDs), in-plane and cross-plane dose profiles, and penumbras for different depths and field sizes of the $6 \mathrm{MV}$ photon beams were measured using ionization chamber and a water tank. The collimator leakages were measured using radiographic films. MMLC and BM were modeled using the EGSnrc-based BEAMnrc Monte Carlo code and above dosimetric parameters were calculated. The energy fluence spectra for the two MLCs were also determined using the BEAMnrc and BEAMDP. Dosimetric parameters of the two MLCs were similar, except for penumbras. Leafside and leaf-end $80-20 \%$ dose penumbras at $10 \mathrm{~cm}$ depth for a $10 \times 10 \mathrm{~cm}^{2}$ field size were 4.8 and $5.1 \mathrm{~mm}$ for MMLC and $5.3 \mathrm{~mm}$ and $6.3 \mathrm{~mm}$ for BM, respectively. Both Radionics MMLC and Elekta BM can be used effectively based on their dosimetric characteristics for stereotactic radiosurgery and radiotherapy, although the former showed slightly sharper dose penumbra especially in the leaf-end direction.

(c) 2012 Associazione Italiana di Fisica Medica. Published by Elsevier Ltd. All rights reserved.
\end{abstract}

\footnotetext{
* Corresponding author. Department of Radiation Physics, 5-613, 610 University Avenue, Toronto, ON M5G 2M9, Canada. Tel.: +1 416 946 4501x5089; fax: +1 4169466566.

E-mail address: james.chow@rmp.uhn.on.ca (J.C.L. Chow).
}

1120-1797/\$ - see front matter @ 2012 Associazione Italiana di Fisica Medica. Published by Elsevier Ltd. All rights reserved. doi:10.1016/j.ejmp.2012.05.001 


\section{Introduction}

Stereotactic radiosurgery (SRS) is routinely used for treatment of a variety of small, well-defined intracranial and extracranial lesions [1]. In the early 1980s, linear accelerators (linacs) were adapted as a competitive equipment to the Gamma Knife unit for stereotactic radiosurgery. Then it rapidly expanded as the modality of choice for treatment of larger cranial tumors using (fractionated) stereotactic radiotherapy (SRT). Linac-based radiosurgical techniques produce highly collimated narrow beams with high spatial precision in dose treatment delivery [2-4].

In the treatment planning of radiosurgery/radiotherapy, a precise dataset of dosimetric parameters such as depth doses, dose profiles and dose output factors (ratio of output at $d_{\text {max }}$ for a given field size to output at $d_{\max }$ for a reference field size of $10 \times 10 \mathrm{~cm}^{2}$ ) is necessary to ensure a precise and high-resolution calculation of dose distribution with very high dose gradient. Beam parameters such as energy, collimation system, field size, source and collimator isocenter distances will affect dose penumbra and hence conformality. Appropriate beam arrangement of generally multiple noncoplanar beams will also help increasing dose gradient and achieving more conformal tumor dose coverage and organs at risk (OAR) avoidance. The relationship between the dose parameters and MLC design can be predicted using Monte Carlo simulation, which is well-known as an accurate calculation method for the dosimetry of external beam radiotherapy. The advantages of using Monte Carlo simulation are (1) a very small voxel size (dose resolution) can be achieved independent to the physical size of the dosimeter; (2) photon energy fluence can be calculated based on the MLC configuration; and (3) the geometric arrangement of the beam and phantom could be set up perfectly in the simulation program. This avoids any experimental human error in positioning the detector in the measurement setup. However, MLCs as beam modifiers in conformal and intensity modulated radiation therapy (IMRT) are still among the most challenging geometries in Monte Carlo simulation. This is due to the very complicated leaf design and their intrafractional movement, making MLC modeling more complex [5,6]. The EGSnrc-based BEAMnrc code is widely used to model linacs. The model of the accelerator is built from a series of independent component modules (CMs) [7,8]. To cope with the complicated and specific design of the MLC, different CMs such as MLCQ [9,10], VARMLC [11], MLCE [12] and DYNVMLC [6] were eventually developed by different groups and coupled to the current BEAMnrc version for MLC modeling.

The aim of this study is to compare dosimetric parameters of two MLCs commonly used in stereotactic radiosurgery/radiotherapy namely, the Radionics MMLC and Elekta Synergy S BM using Monte Carlo simulations with verifications through dosimetry measurements.

\section{Materials and methods}

\section{MMLC and BM}

Radionics MMLC is a micro-MLC that is attached to the linac's head as an accessory (Fig. 1a). It contains 31 pairs of independent tungsten leaves, with a maximum field size of
$10 \times 12 \mathrm{~cm}^{2}, 7 \mathrm{~cm}$ leaf height and $33 \mathrm{~cm}$ isocentre clearance. Projected to the isocentre, each leaf has a $3.8 \mathrm{~mm}$ width. It has a straight leaf-end with tongue-and-groove design. The edges of MLC leaves should ideally be directed toward the source (focusing), independent of the leaf position to cause smallest penumbra. In "single focusing" the leaves move perpendicular to the beam central axis but in "double focusing" they move along an arc centered on the X-ray target $[13,14]$. Radionics MMLC is designed to be double focused. The installation system makes placement and removal of the unit easy.

Elekta BM is enclosed inside the head of the Synergy $S$ linac. The BM is a commercial name of the MLC used in the Elekta Synergy $\mathrm{S}$ linac. It contains 80 independent leaves with two banks. The leave height is equal to $7.5 \mathrm{~cm}$ and the maximum field size is $16 \times 21 \mathrm{~cm}^{2}$. The leave is made of tungsten alloy consisting of tungsten, nickel and iron. Unlike MMLC, the leaves travel linearly and not in an arc. The BM leaves can perform interdigitation (i.e. tips of neighboring leaves on opposed MLC banks are allowed to pass one another) and have $4 \mathrm{~mm}$ width at the isocenter. Since there is no moveable backup jaw, the treatment field is only defined by the leaves (Fig. 1b). The leaf-ends of the $\mathrm{BM}$ are rounded with radius of curvature equal to $12 \mathrm{~cm}$, which is different from the MMLC. Moreover, tongue-andgroove design cannot be found in BM as it uses air gaps of about $0.1 \mathrm{~mm}$ between adjacent leaves to minimize movement friction [15].

A summary of geometric and dosimetric parameters of MMLC and BM can be found in Table 1. As it is seen from this table, the BM has larger maximum field size, number of MLC
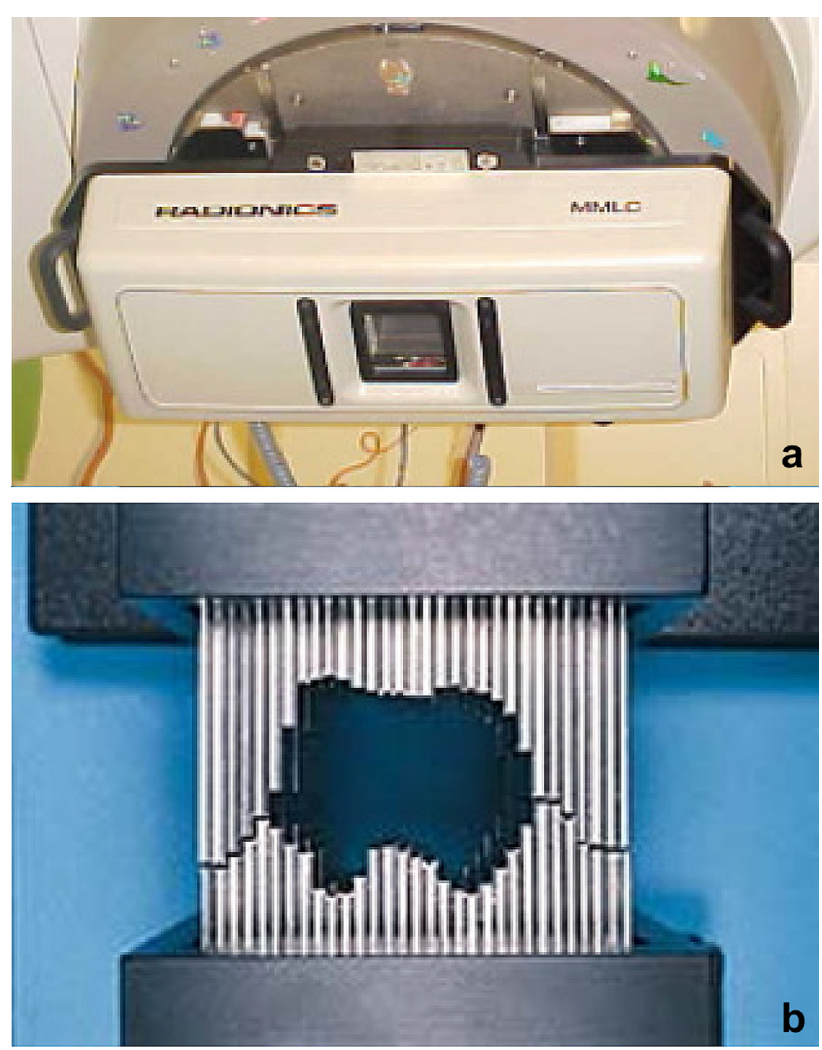

Figure 1 Radionics MMLC (a) and BM leaf bank (b). 
Table 1 Geometric and dosimetric characteristics of the MMLC and BM.

\begin{tabular}{lll}
\hline Parameter & Radionics MMLC & Beam modulator \\
\hline Maximum field size & $10 \times 12 \mathrm{~cm}^{2}$ & $16 \times 21 \mathrm{~cm}^{2}$ \\
Number of leaves & 31 pairs & 40 pairs \\
Leaf thickness at isocenter & $3.8 \mathrm{~mm}$ & $4.0 \mathrm{~mm}$ \\
Resolution & $0.1 \times 3.75 \mathrm{~mm}^{2}$ & $0.1 \times 4.0 \mathrm{~mm}^{2}$ \\
Leaf Height & $7 \mathrm{~cm}$ & $7.5 \mathrm{~cm}$ \\
Leakage $\%$ & Maximum $=1.2 ;$ Average $=0.9$ & Maximum $=1.3 ;$ Average $=1.0$ \\
Isocentric clearance & $33 \mathrm{~cm}$ & $45.2 \mathrm{~cm}$ \\
Penumbra $(80-20 \%) 10 \times 10 \mathrm{~cm}^{2}$ & $3.6 \mathrm{~mm}$ and $3.8 \mathrm{~mm}$ & $3.9 \mathrm{~mm}$ and $4.7 \mathrm{~mm}$ \\
$\quad$ field at $d_{\text {max leaf-side and leaf-ends }}$ & & $5.3 \mathrm{~mm}$ and $6.3 \mathrm{~mm}$ \\
Penumbra $(80-20 \%) 10 \times 10 \mathrm{~cm}^{2}$ & $4.8 \mathrm{~mm}$ and $5.1 \mathrm{~mm}$ & \\
$\quad$ field at $10 \mathrm{~cm}$ depth leaf-side and leaf-ends & & \\
\hline
\end{tabular}

leaves and slightly larger leaf thickness than the MMLC. The isocentric clearance of the $B M$ is $45.2 \mathrm{~cm}$, which is also larger than that of the MMLC $(33 \mathrm{~cm})$.

\section{Monte Carlo simulation}

The EGSnrc-based BEAMnrc code was used to model the $M M L C$ and BM for a $6 \mathrm{MV}$ photon beam $[7,8]$. Figure 2 shows schematic diagram of $Y-Z$ view across the leaves of the BM (perpendicular to its leaf movement) of the Synergy $S$ treatment head with the associated components including the MMLC as accessory. Different CMs were used in the BEAMnrc/EGSnrc to model different sections of the linac head. The only field-defining part of the linac is the BM.

An incident electron beam on the target with a peak energy of $6.5 \mathrm{MeV}$, and a Gaussian intensity distribution with a full width at half maximum (FWHM) of $0.11 \mathrm{~cm}$ in displacement and $0.5 \mathrm{MeV}$ in energy was determined by matching simulated PDD and beam profiles with measured data [16]. The electron energy cut-off was set to $0.7 \mathrm{MeV}$, the photon energy cut-off to $0.01 \mathrm{MeV}$ and there was no

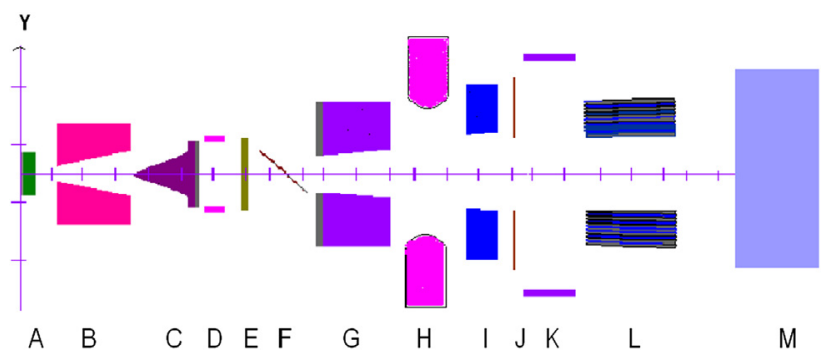

Figure 2 Schematic diagram of Elekta "Synergy $S$ " treatment head with Radionics MMLC attached as an accessory. It shows the $Y Z$ view across the leaves of the Radionics MMLC (perpendicular to its leaf movement). We used, respectively, SLABS CM for the target $(A)$, CONS3R CM for the primary collimator $(B)$, FLATFILT CM for the flattening filter (C), CHAMBER CM for the monitor (D), SLABS CM for the back scatter plate (E), MIRROR CM for the mirror $(F)$, PYRAMIDS CM for the Millstone collimator $(G)$, MLCE CM for the Beam Modulator $(H)$, JAWS for the fixed outer diaphragm (I) APPLICAT CM for clamp (J), CONS3R CM for steel ring $(K)$ and VARMLC for Radionics MMLC (L) followed by CHAMBER CM $(M)$ for the DOSXYZnrc phantom, depending on the simulation. photon interaction forcing or Rayleigh scattering. Range rejection and directional bremsstrahlung splitting (DBS) were enabled as variance reductions. ESAVE is a parameter in the EGSnrc code, and was set to $2 \mathrm{MeV}$ for the range rejection. It means that the history of every electron with a kinetic energy lower than $2 \mathrm{MeV}$ terminates and deposits all of its energy in the current region, if it fails to reach the nearest boundary. This value for ESAVE makes simulation 2-3 times shorter and ignores only $0.1 \%$ of photons [17]. The VARMLC CM was used to model the MMLC, while the MLCE CM was used to model the BM. The VARMLC is similar to MLC and MLCQ CMs but it can also simulate the tongueand-groove which connects adjacent leaves, the screws at the top and bottom, which are used to open and close leaves and the air gap which connects the leaves. The MLCE modeled the BM including the tilt and step design of the leaf. The BEAMDP [18] as an important program for processing phase-space files and a general-purpose utility was used to derive energy spectra for photon beams as well as contaminating electrons and positrons at the phantom surface.

The DOSXYZnrc code [19] was applied to simulate particle transport in a water phantom. The information scored in the phase-space plane at source-surface distance $(S S D=90 \mathrm{~cm})$ were used for the dose measurements at isocentre level and $10 \mathrm{~cm}$ depth. Also Phantom CM in BEAMnrc with phase-space at SSD $=98.5 \mathrm{~cm}$ was used to calculate PDDs. These parameters were also calculated for different field sizes, SSDs and depths. The voxel dimensions were adapted according to the expected dose gradients; e.g. $0.5-1 \mathrm{~cm}$ in the flat region of the profiles and $0.1 \mathrm{~cm}$ in the penumbra region.

Number of particle histories in the phase space depends on the field size [9]. To obtain acceptable statistical variances less than $0.5 \%$, the particles were recycled more than 100 times so that one billion particles were gathered together in the phase space for $10 \times 10 \mathrm{~cm}^{2}$ field size. The statistical variances better than $0.5 \%$ were therefore achieved for all depth doses and off-axes dose profiles.

\section{Dosimetric measurement and Monte Carlo verification}

Ionization chambers model CC13 (Scanditronics Wellhofer) was used for dose measurements. It is a water-proof 
chamber with air cavity volume of $0.13 \mathrm{~cm}^{3}$ and $3.0 \mathrm{~mm}$ cavity radius. Three dosimetric parameters, PDDs, dose profiles (in-plane and cross-plane) and penumbras were measured in a Wellhofer water tank under different setup conditions of field sizes and depths. In this study, since it is impossible to set a $10 \times 10 \mathrm{~cm}^{2}$ symmetrical reference field on the BM due to the finite leaf width, the reference field was indeed selected as $9.6 \times 10.4 \mathrm{~cm}^{2}$. However, in this study we refer to that field size as $10 \times 10 \mathrm{~cm}^{2}$.

PDDs were obtained for different field sizes at $\mathrm{SSD}=98.5 \mathrm{~cm}$. Dose profiles were measured for 3 field sizes $\left(4 \times 4,8 \times 8\right.$ and $\left.10 \times 10 \mathrm{~cm}^{2}\right)$ at $10 \mathrm{~cm}$ depth and $90 \mathrm{~cm}$ SSD. Dose profiles were also measured for $10 \times 10 \mathrm{~cm}^{2}$ field size at $1.5,5$ and $10 \mathrm{~cm}$ depths at $98.5,95$ and $90 \mathrm{~cm}$ SSD, respectively. Lateral dose profiles for different field sizes and depths were obtained in both leafside and leaf-end directions (the direction perpendicular to the MLC movement) in the water phantom. All measured results were compared with the corresponding Monte Carlo results. Dose penumbras $(80-20 \%)$ were measured from the beam profiles under different setup conditions of field sizes and depths. The leakage and transmission measurements were done using film at depth of $5 \mathrm{~cm}$ of solid water and $\mathrm{SSD}=95 \mathrm{~cm}$ providing sufficient layers of solid water to take into account backscatter factor.

It was found that deviation between the measured and Monte Carlo depth dose results were smaller than $\pm 0.5 \%$, while those of the beam profiles were smaller than $\pm 1 \%$ or $1 \mathrm{~mm}$. These deviations were calculated in percentage differences between the Monte Carlo and measured results, and were typically accepted as a satisfaction of the Monte Carlo dosimetric verification [20,21]. Since this study is focused on the dosimetric comparison between the MMLC and $\mathrm{BM}$, corresponding results of Monte Carlo verification are not shown here due to the limited space.

\section{Results and discussion}

Energy fluence distribution of the photon as well as electron and positron produced by the MMLC and BM for a $10 \times 10 \mathrm{~cm}^{2}$ field and nominal $6 \mathrm{MV}$ photon beam is shown in Fig. 3. Percentage depth doses (PDDs) of MMLC and BM are shown in Fig. 4 for (a) $4 \times 4 \mathrm{~cm}^{2}$ and (b) $10 \times 10 \mathrm{~cm}^{2}$ field sizes. In-plane (leaf-side) dose profiles for $4 \times 4 \mathrm{~cm}^{2}$ and $10 \times 10 \mathrm{~cm}^{2}$ field sizes are shown in Figs. $5 \mathrm{a}$ and $6 \mathrm{a}$, respectively. Corresponding cross-plane (leaf-end) dose profiles are shown in Figs. $5 \mathrm{~b}$ and $6 \mathrm{~b}$. Figure $7 \mathrm{a}$ and $\mathrm{b}$ shows the measured leaf-side and leaf-end penumbras (80-20\%) for the MMLC and BM for different field sizes at a depth of $10 \mathrm{~cm}$, while Fig. $8 \mathrm{a}$ and $\mathrm{b}$ shows the penumbras for $10 \times 10 \mathrm{~cm}^{2}$ field at different depths. Both the beam profiles and penumbras were measured using the CC13 ionization chamber. The radiation leakage of the MMLC and $\mathrm{BM}$ is shown in Fig. 9 measured using film.

\section{Energy fluence spectrum}

Using the BEAMDP code, the energy fluence distribution of the photon beam and also contaminant electrons and positrons were calculated. Figure 3 shows the energy spectra for the $6 \mathrm{MV}$ photon beams and also electrons and

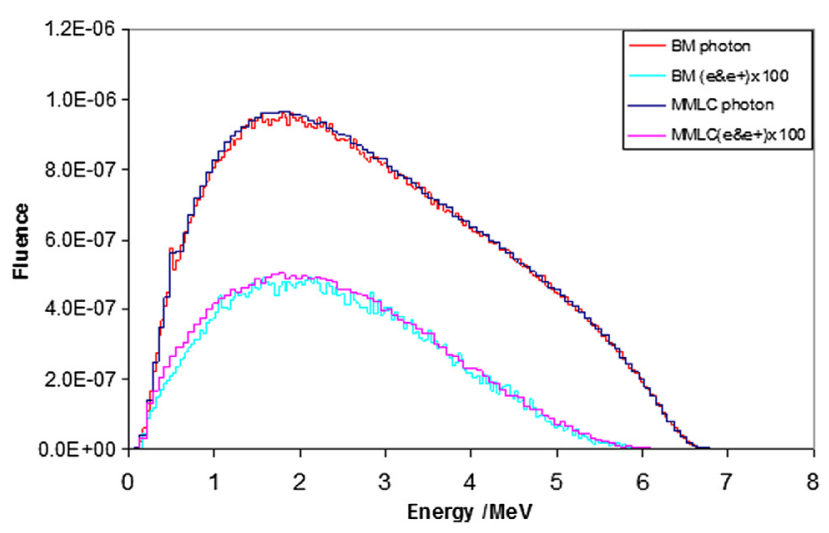

Figure 3 Photon, electron and positron energy fluence distribution comparison between MMLC and BM for a $6 \mathrm{MV}$ photon beam and $10 \times 10 \mathrm{~cm}^{2}$ field size.

positrons per $\mathrm{MeV}$ per incident particle for a $10 \times 10 \mathrm{~cm}^{2}$ field at $S S D=90 \mathrm{~cm}$ on the phantom surface in air. The spectra of contaminant electrons for both the MMLC and BM have similar shape to those of primary photons. This is similar to what has been reported earlier for nine photon beams from different commercial medical linacs of three
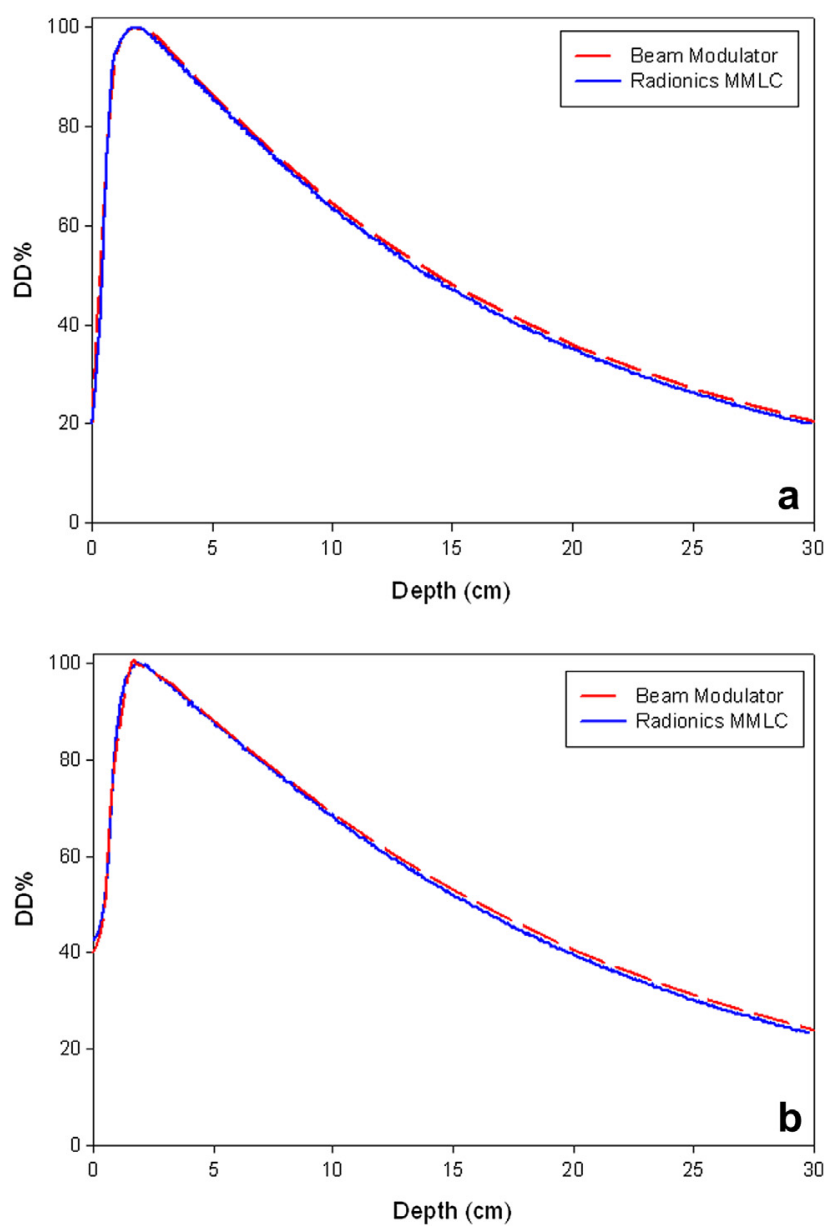

Figure 4 MMLC and BM measured PDDs for (a) $4 \times 4 \mathrm{~cm}^{2}$; and (b) $10 \times 10 \mathrm{~cm}^{2}$ field sizes. 

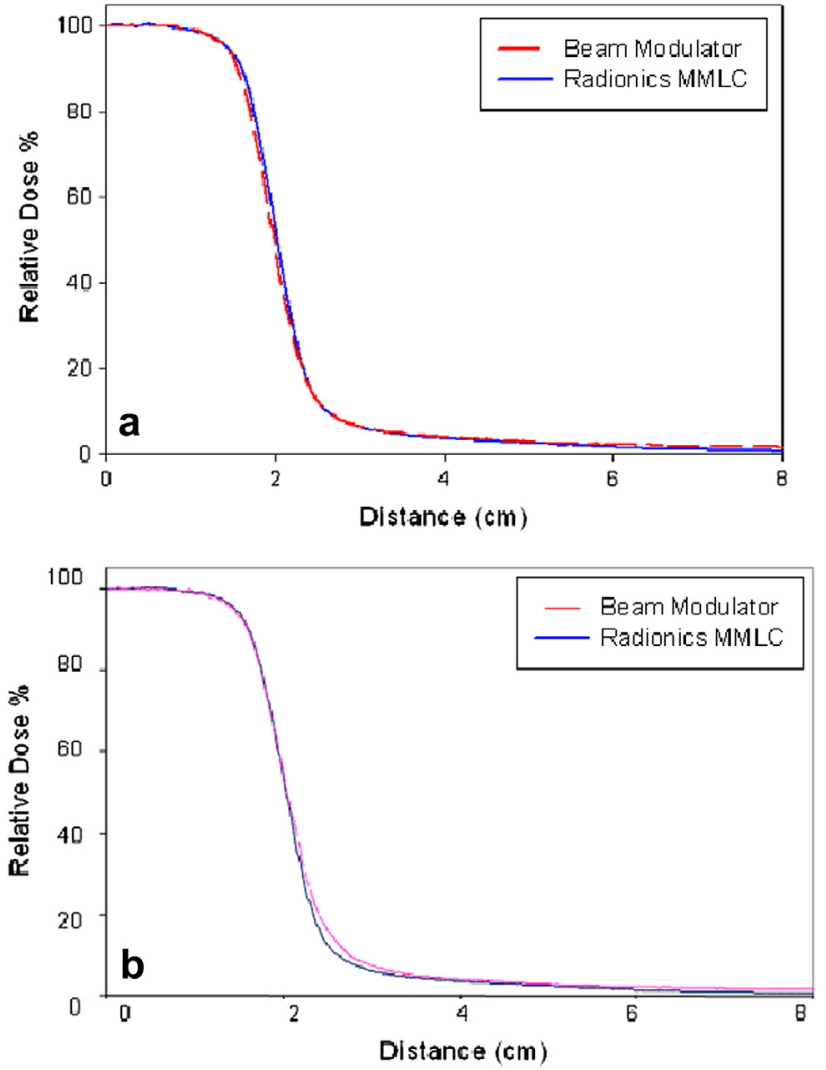

Figure $5 \quad M M L C$ and BM dose profiles for a $4 \times 4 \mathrm{~cm}^{2}$ field for (a) in-plane and (b) cross-plane directions.

major manufacturers [22]. Energy spectra of incident photon and also positron and electron peak are at about $2 \mathrm{MeV}$. Electron and positron spectra are multiplied by 100 to be shown on the same figure as for the photon spectrum. The counts per incident electron on the target and per unit energy for photons are about 200 times more than those of contaminant electrons and positrons. In Fig. 3, it can be seen that the deviation of the energy fluence between MMLC and BM is not significant based on the Monte Carlo simulation results.

\section{PDD, dose profile and penumbra}

Photon beam PDDs (6 MV) for MMLC and BM are fairly similar, as it is shown in Fig. 4, where the latter is only slightly more penetrative for both small $\left(\mathrm{PDD}_{10 \mathrm{~cm}}=63.94 \%\right.$ vs. $63.8 \%$ for Fig. $4 \mathrm{a})$ and large $\left(\mathrm{PDD}_{10 \mathrm{~cm}}=67.8 \%\right.$ vs. $67.7 \%$ for Fig. 4b) field sizes. This can be attributed to larger BM distance to point of measurements compared to MMLC. Dose profiles for $4 \times 4$ and $10 \times 10 \mathrm{~cm}^{2}$ field sizes are shown in Figs. 5 and 6, respectively. In-plane (leaf-side) dose profiles are shown in Figs. $5 \mathrm{a}$ and $6 \mathrm{a}$, it is seen that the MMLC has a smaller penumbra than BM for both field sizes. The difference is due to smaller distance of MMLC compared to BM. This is true and even more pronounced in the cross-plane (leaf-end) direction as it is shown in Figs. 5b and $6 \mathrm{~b}$. The reason is rounded leaf-ends for $\mathrm{BM}$ as well as its larger distance. Further details of the penumbra width measurements $(80-20 \%)$ can be found in Figs. 7 and 8 .
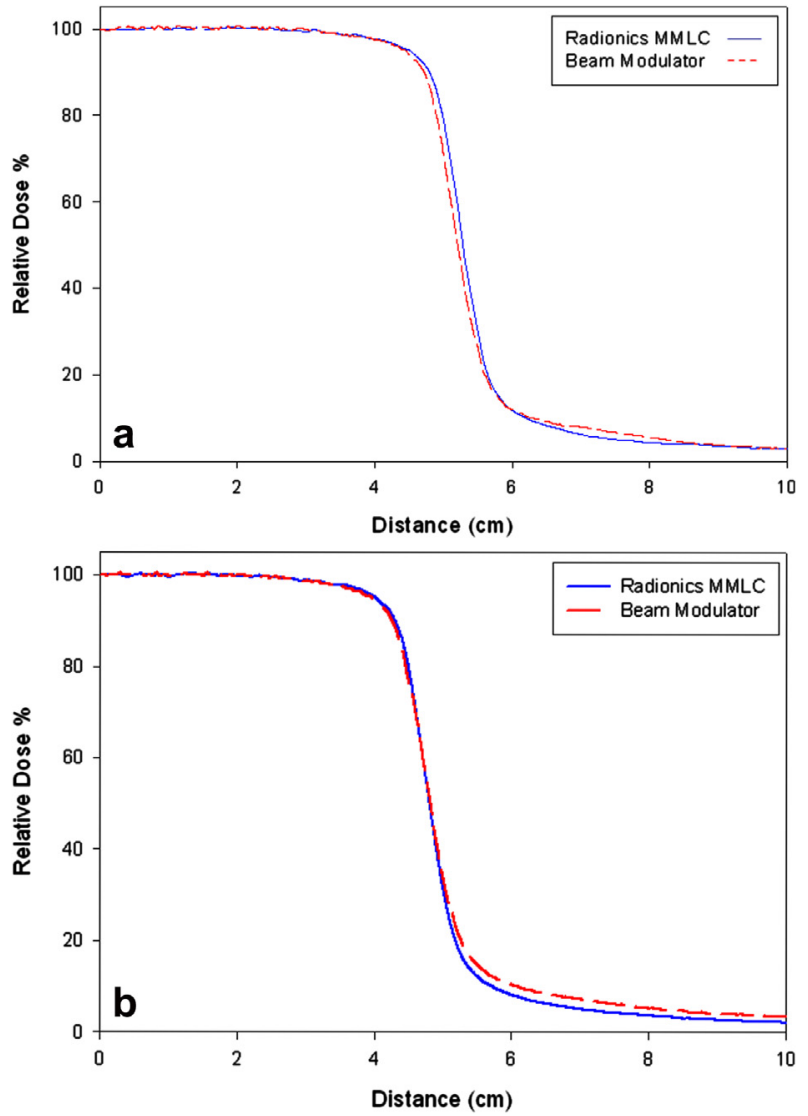

Figure 6 MMLC and BM dose profiles for a to $10 \times 10 \mathrm{~cm}^{2}$ field for (a) in-plane and (b) cross-plane directions.

Figure $7 \mathrm{a}$ and $\mathrm{b}$ shows the measured leaf-side and leaf-end dose profiles for $4 \times 4,8 \times 8$ and $10 \times 10 \mathrm{~cm}^{2}$ field sizes at $10 \mathrm{~cm}$ depth and SSD $=90$. It is found that the penumbra width is generally smaller for the flat-end than for the round-end designed MLC [23]. In Fig. 7, as expected, the penumbra width is larger (worsen) with increasing field size. Take the MMLC as an example, the $80-20 \%$ penumbra width under the above setup conditions is ranged from 4.1 to $5.1 \mathrm{~mm}$ for the leaf-ends and $4.0-4.8 \mathrm{~mm}$ for the leafsides for different field sizes from $4 \times 4$ to $10 \times 10 \mathrm{~cm}^{2}$. Variations of the dose penumbras with depth $(1.5,5$ and $10 \mathrm{~cm}$ ) for a $10 \times 10 \mathrm{~cm}^{2}$ field are shown in Fig. 8 for both the leaf-side (a) and leaf-end (b) directions. The corresponding values for penumbras of the MMLC at depths of 1.5 and $5 \mathrm{~cm}$ are 3.6 and $4.2 \mathrm{~mm}$ for the leaf-sides and 3.8 and $4.5 \mathrm{~mm}$ for the leaf-ends. The leaf-end penumbras are up to $0.3 \mathrm{~mm}$ more than the leaf-side. Improving the penumbra for leaf-sides/ends in the gun-target direction, is possibly due to impact of focal spot size on penumbra [24]. Also the penumbra will increase due to the volume averaging and the non-water equivalence of ionization chamber. This is due to the higher range of electrons in air compared to water. In this work, method of Dawson was applied for correction of ionization chamber measured penumbra. It is a standard correction method to eliminate the dependence of the penumbra measurements on the inside diameter of the ionization chamber [25]. The method is based on extrapolation of ionization chamber data to 

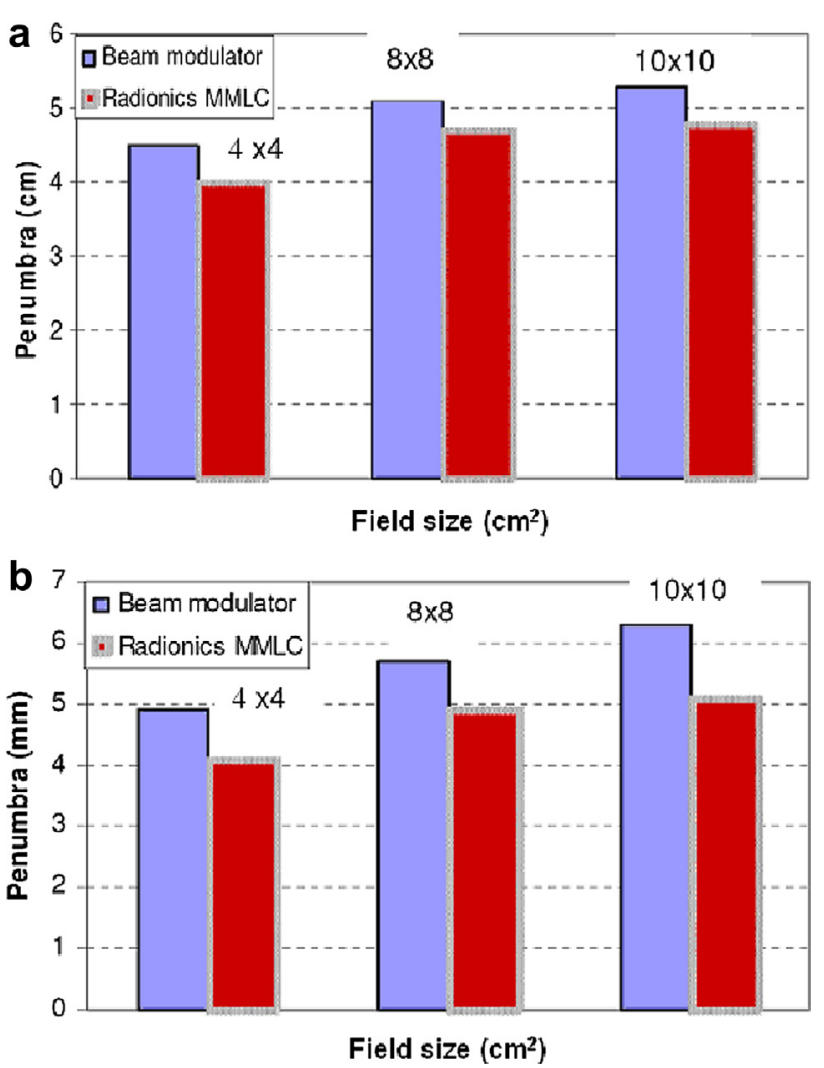

Figure $7 \quad M M L C$ and BM measured leaf-side (a) and leaf-end (b) penumbras (80-20\%) for different field sizes at $10 \mathrm{~cm}$ depth.
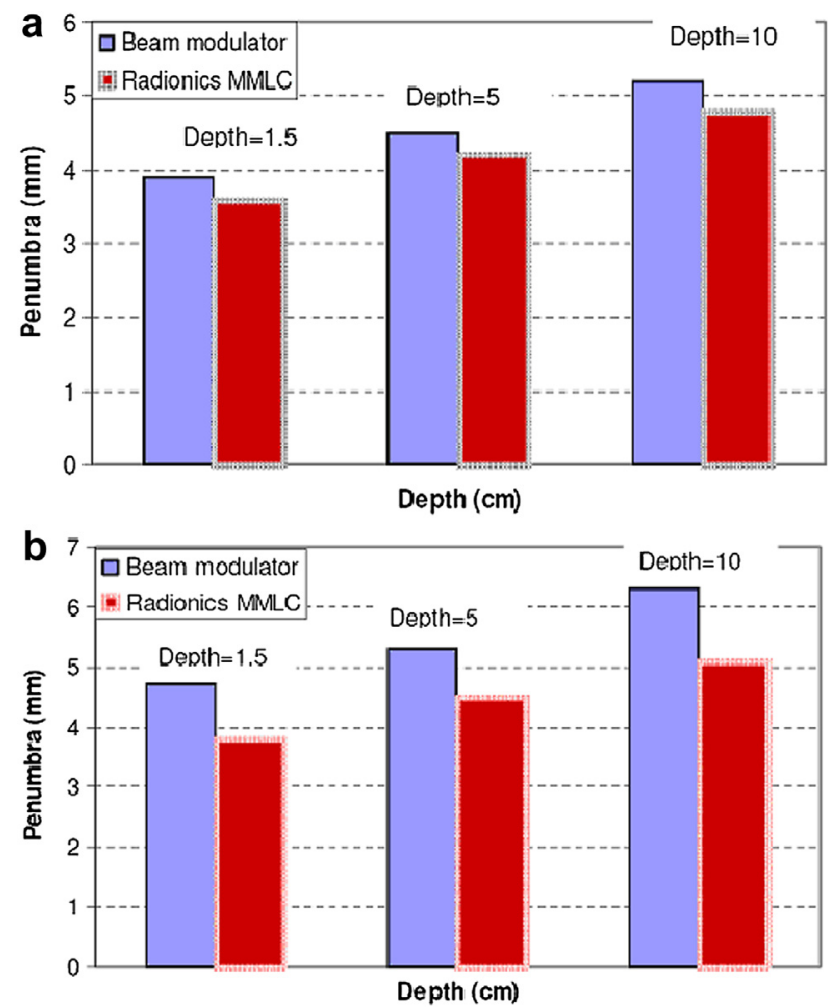

Figure 8 MMLC and BM measured leaf-side (a) and leaf-end (b) penumbras $(80-20 \%)$ for a $10 \times 10 \mathrm{~cm}^{2}$ field at $\mathrm{SAD}=100 \mathrm{~cm}$ at different depths.

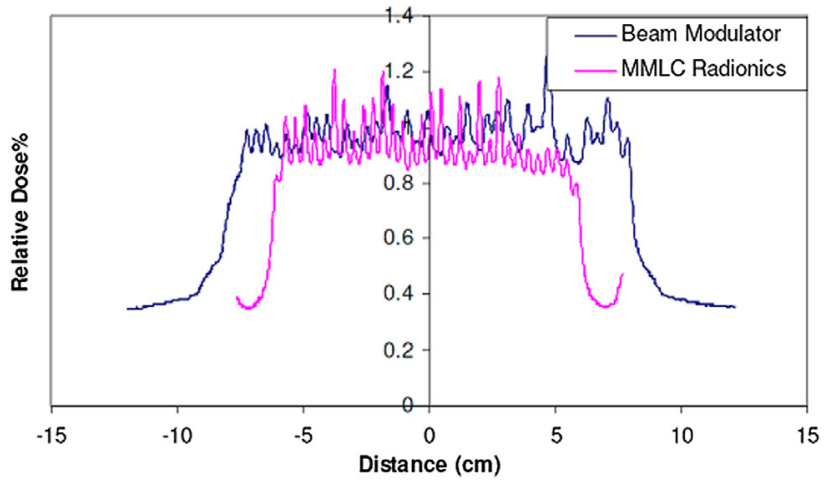

Figure 9 Leakage comparison by film measurement at a depth of $5 \mathrm{~cm}$ and $\mathrm{SSD}=95 \mathrm{~cm}$ normalized to $10 \mathrm{~cm}$ square open field.

zero diameters for accurate results. In Figs. 7 and 8, it is seen that MMLC has a smaller penumbras than those of BM for all combinations of field size, depth, leaf-side and leafend.

\section{Leakage and transmission}

Figure 9 shows the leaf-side leakage measurement results using film for the MMLC and BM. It is seen that both the leakage and transmission for the MMLC and BM are similar. The maximum percentages of the leakage for the MMLC and $\mathrm{BM}$ are $1.2 \%$ and $1.3 \%$, respectively, while the average percentage of leakage for the MMLC and BM are $0.9 \%$ and $1 \%$, respectively, as shown in Table 1 . Moreover, both MMLC and BM have leaf transmission smaller than $1.4 \%$ as shown in Fig. 9.

\section{Conclusions}

Radionics MMLC and BM associated with the Elekta "Synergy S" linac were successfully modeled for Monte Carlo simulations using the BEAMnrc code. Beam characteristics such as PDDs, beam profiles, penumbra widths, leaf leakage and transmission for both MLCs were determined by Monte Carlo simulations and measurements with different field sizes and depths using the $6 \mathrm{MV}$ photon beams. Our results from both Monte Carlo simulations and measurements showed that both MLCs have comparable beam characteristics such as energy fluence spectrum, PDD, beam profile, penumbra width, leakage and transmission. Although the MMLC has a smaller penumbra than that of the BM for different combinations of field sizes and depths, BM has the advantages of producing considerably larger field at isocenter and having a greater isocenter clearance compared to other MLCs. Monte Carlo and measured results in this study should help radiotherapy staff to appreciate dependence of dosimetry on the MLC design and configuration.

\section{Conflict of interest}

Authors have no conflicts of interest to report. 


\section{Acknowledgments}

This work was supported by the Radiation Medicine Program, Princess Margaret Hospital (Canada) and Tehran University of Medical Sciences (Iran). The authors appreciate help from Dr. Blake Walters for support in the BEAMnrc code (National Research Council of Canada).

\section{References}

[1] Heydarian M, Hoban PW, Beddoe AH. A comparison of dosimetry techniques in stereotactic radiosurgery. Phys Med Biol 1996;41:93-110.

[2] Slotman B, Solberg T, Verellen D. Extracranial stereotactic radiotherapy and radiosurgery. New York: Talor \& Francis Group; 2006.

[3] Liu H, Verhaegen F, Dong L. A method to simulate Dynamic Multileaf Collimators using Monte Carlo techniques for intensity modulated radiation therapy. Phys Med Biol 2001;46: 2283-98.

[4] Belec J, Patrocinnio H, Verhaegen F. Development of a Monte Carlo model for the Brainlab microMLC. Phys Med Biol 2005; 50:787-99.

[5] Verhaegen F, Seuntjens J. Monte Carlo modeling of external radiotherapy photon beams. Phys Med Biol 2003;48:R107-64.

[6] Heath $H$, Seuntjens J. Development and validation of a BEAMnrc component module for accurate Monte Carlo modeling of the Varian dynamic Millennium multileaf collimator. Phys Med Biol 2003;48:4045-63.

[7] Rogers DWO, Faddegon BA, Ding GX, Ma CM, We J, Mackie TR. BEAM: a Monte Carlo code to simulate radiotherapy treatment units. Med Phys 1995;22:503-24.

[8] Rogers DWO, Ma CM, Walters B, Ding GX, Sheikh-Bagheri D, Zhang G. BEAMnrc users manual. Report PIRS-0509(A) revK. NRCC; 2002.

[9] De Vlamynck K, Palmans H, Verhaegen F, De Wagter C, De Neve W, Thierens H. Dose measurements compared with Monte Carlo simulations of narrow 6 MV multileaf collimator shaped photon beams. Med Phys 1999;26:1874-82.

[10] Palmans H, Verhaegen F, Buffa FM, Mubata C. Considerations for modeling MLCs with Monte Carlo techniques. R162 Topical Review. In: Proceedings of the 13th international conference on the use of computers in radiation therapy. Heidelberg Berlin: Springer; 2000. p. 458-60.
[11] Monte Carlo simulations for multileaf-collimator leaves: design and dosimetry. In: Kapur A, Ma CM, Boyer AL, editors. World congress on medical physics and biomedical engineering, July 23-28;Chicago, Illinois. Med Phys 2000;27:1410 [Abstract].

[12] Van de Walle J, Martens C, Reynaert N, Palmans H, Coghe M, De Neve W, et al. Monte Carlo model of the Elekta SLiplus accelerator: validation of a new MLC component module in BEAM for a 6 MV beam. Phys Med Biol 2003;48:371-85.

[13] Khan FM. The physics of radiation therapy. Baltimore, USA: Williams \& Wilkins; 1984.

[14] AAPM Radiation Therapy Committee Task Group No. 50, report no. 72, 2001.

[15] Heydarian M, Asnaashari K, Allahverdi M, Jaffray DA. Dosimetric evaluation of a dedicated stereotactic linear accelerator using measurement and Monte Carlo simulation. Med Phys 2008;35:3943-54.

[16] Sheikh-Bagheri D, Rogers DWO. Sensitivity of megavoltage photon beam Monte Carlo simulations to electron beam parameters. Med Phys 2002;29:379-90.

[17] Sheikh-Bagheri D, Rogers DWO, Ross CK, Seuntjens JP. Comparison of measured and Monte Carlo calculated dose distributions from the NRC linac. Med Phys 2000;27:2256-66.

[18] Ma CM, Rogers DWO. BEAMDP users manual. NRCC Report PIRS0509C. Ottawa: NRC; 1995.

[19] Ma CM, Reckwerdt P, Holmes M, Rogers DWO, Geiser B, Walters B. DOSXYZ users manual. PIRS-0509b. National Research Council of Canada; 1995.

[20] Spezi E, Lewis DG, Smith CW. Monte Carlo simulation and dosimetric verification of radiotherapy beam modifiers. Phys Med Biol 2001;46:3007-29.

[21] Li JS, Pawlicki T, Deng J, Jiang SB, Mok E, Ma CM. Validation of a Monte Carlo dose calculation tool for radiotherapy treatment planning. Phys Med Biol 2000;45:2969-85.

[22] Sheikh-Bagheri D, Rogers DWO. Monte Carlo calculation of nine megavoltage photon beam spectra using the BEAM code. Med Phys 2002;29:391-402.

[23] Arnfield MR, Qiuwen WU, Tong S, Mohan R. Dosimetric validation for multileaf collimator-based intensity-modulated radiotherapy: a review. Med Dosim 2001;26:179-88.

[24] Patel I, Glendinning AG, Kirby MC. Dosimetric characteristics of the Elekta Beam Modulator. Phys Med Biol 2005;50: 5479-92.

[25] Dawson DJ, Harper JM, Akinradewo AC. Analysis of physical parameters associated with the measurement of high-energy x-ray penumbra. Med Phys 1984;11:491-7. 